\title{
Local Hopf Bifurcation in a Competitive Model of Market Structure with Consumptive Delays
}

\author{
Xuhui Li \\ Antai College of Economics and Management, Shanghai Jiao Tong University, Shanghai 200030, China \\ Correspondence should be addressed to Xuhui Li; xuhuiliantai@163.com
}

Received 9 November 2013; Accepted 4 April 2014; Published 4 May 2014

Academic Editor: Mehmet Sezer

Copyright ( 2014 Xuhui Li. This is an open access article distributed under the Creative Commons Attribution License, which permits unrestricted use, distribution, and reproduction in any medium, provided the original work is properly cited.

A competitive model of market structure with consumptive delays is considered. The local stability of the positive equilibrium and the existence of local Hopf bifurcation are investigated by analyzing the distribution of the roots of the associated characteristic equation. The explicit formulas determining the stability and other properties of bifurcating periodic solutions are derived by using normal form theory and center manifold argument. Finally, numerical simulations are given to support the analytical results.

\section{Introduction}

The Lotka-Volterra predator-prey model is proposed by Lotka and Volterra to describe the dynamics between populations in ecology. And it has been extensively studied by many authors [1-7]. Recently, the Lotka-Volterra predator-prey model has been used in economics by many scholars [8-11]. In [8], Brander and Taylor proposed a general equilibrium model of renewable resource and population dynamics related to the Lotka-Volterra predator-prey model. They applied the model to the rise and fall of Easter Island and showed that plausible parameter values generate a "feast and famine" pattern of cyclical adjustment in population and resource stocks. In [9], Delfino and Simmons investigated the links between the health structure of the population and the productive system of an economy which is subject to infectious disease by combining the Lotka-Volterra model with Solow-Swan growth model. They analyzed the local dynamics and found that the epidemiological-economic stationary state is locally stable and an attractor for a wide range of initial conditions. In [10], Farmer derived a simple nonequilibrium model for price formation. He applied the model to several commonly used trading strategies and discussed how the model can be used to understand the long term evolution of financial markets. In [11], Kong analyzed the evolution of market structure with
Lotka-Volterra model. And a model of market structure was established by simulating the relations of product in market:

$$
\begin{aligned}
& \frac{d x(t)}{d t}=r_{1} x(t)\left[1-\frac{x(t)}{N_{1}}-\sigma_{12} \frac{y(t)}{N_{2}}\right], \\
& \frac{d y(t)}{d t}=r_{2} y(t)\left[1-\frac{y(t)}{N_{2}}-\sigma_{21} \frac{x(t)}{N_{1}}\right],
\end{aligned}
$$

where $x(t)$ denotes the output of the product $X$ at time $t . y(t)$ denotes the output of the product $Y$ at time $t . r_{1}$ and $r_{2}$ denote the growth rates of the products $X$ and $Y$, respectively. $N_{1}$ and $\mathrm{N}_{2}$ denote the production scale of the products $X$ and $Y$, respectively. $\sigma_{12}$ and $\sigma_{21}$ are the competition rates between the products $X$ and $Y$. In system (1), $X$ and $Y$ are products of the same type but produced by different manufacturers. Kong [11] obtained the conditions for the market structure coming into being by analyzing the stability of system (1).

It is well known that time delays are universal in the market structure due to the consumption, competition, or other reasons. Therefore, it is necessary to incorporate time 
delays into system (1). Based on this consideration, we consider the following model with time delays:

$$
\begin{aligned}
& \frac{d x(t)}{d t}=r_{1} x(t)\left[1-\frac{x\left(t-\tau_{1}\right)}{N_{1}}-\sigma_{12} \frac{y(t)}{N_{2}}\right], \\
& \frac{d y(t)}{d t}=r_{2} y(t)\left[1-\frac{y\left(t-\tau_{2}\right)}{N_{2}}-\sigma_{21} \frac{x(t)}{N_{1}}\right],
\end{aligned}
$$

where $\tau_{1}$ and $\tau_{2}$ are the consumption delays of the product $X$ and $Y$, respectively. All the parameters in system (2) have the same meanings as in (1) and all of them are assumed to be positive.

This paper is organized as follows. In Section 2, we analyze the local stability of the positive equilibrium and the existence of the local Hopf bifurcation. In Section 3, we determine the direction of the Hopf bifurcation and the stability of the bifurcating periodic solutions by using normal form theory and center manifold argument. To support the analytical results, numerical simulations are included at last.

\section{Local Stability of the Positive Equilibrium and Existence of the Hopf Bifurcation}

Considering the economical significance of the system (2), we are interested only in the positive equilibrium of (2).

It is not difficult to verify that if conditions $\left(H_{1}\right): 0<$ $\sigma_{12}<1$ and $\left(H_{2}\right): 0<\sigma_{21}<1$ hold, system (2) has a unique positive equilibrium $E_{*}\left(x_{*}, y_{*}\right)$, where

$$
x_{*}=\frac{\left(1-\sigma_{12}\right) N_{1}}{\left(1-\sigma_{21}\right) N_{2}} y_{*}, \quad y_{*}=\frac{N_{2}\left(1-\sigma_{21}\right)}{1-\sigma_{12} \sigma_{21}} .
$$

Let $u_{1}(t)=x(t)-x_{*}, u_{2}(t)=y(t)-y_{*}$, and $u_{3}(t)=y_{2}(t)-$ $y_{2}^{*}$. We still denote $u_{1}(t), u_{2}(t)$ by $x(t), y(t)$, respectively. Then system (2) can be transformed to the following form:

$$
\begin{aligned}
\frac{d x(t)}{d t}= & a_{12} y(t)+b_{11} x\left(t-\tau_{1}\right) \\
& +a_{13} x(t) y(t)+a_{14} x(t) x\left(t-\tau_{1}\right), \\
\frac{d y(t)}{d t}= & a_{21} x(t)+c_{22} y\left(t-\tau_{2}\right) \\
& +a_{23} x(t) y(t)+a_{24} y(t) y\left(t-\tau_{2}\right),
\end{aligned}
$$

where

$$
\begin{aligned}
a_{12}=-\frac{r_{1} \sigma_{12}}{N_{2}} x_{*}, & b_{11}=-\frac{r_{1}}{N_{1}} x_{*}, \\
a_{21}=-\frac{r_{2} \sigma_{21}}{N_{1}} y_{*}, & c_{22}=-\frac{r_{2}}{N_{2}} y_{*}, \\
a_{13}=-\frac{r_{1} \sigma_{12}}{N_{2}}, & a_{14}=-\frac{r_{1}}{N_{1}}, \\
a_{23}=-\frac{r_{2} \sigma_{21}}{N_{1}}, & a_{24}=-\frac{r_{2}}{N_{2}} .
\end{aligned}
$$

The linearized system of system (4) is

$$
\begin{aligned}
& \frac{d x(t)}{d t}=a_{12} y(t)+b_{11} x\left(t-\tau_{1}\right), \\
& \frac{d y(t)}{d t}=a_{21} x(t)+c_{22} y\left(t-\tau_{2}\right) .
\end{aligned}
$$

The characteristic equation of system (6) at the positive equilibrium is

$$
\lambda^{2}+A+B \lambda e^{-\lambda \tau_{1}}+C \lambda e^{-\lambda \tau_{2}}+D e^{-\lambda\left(\tau_{1}+\tau_{2}\right)}=0,
$$

where

$$
\begin{gathered}
A=-a_{12} a_{21}<0, \quad B=-b_{11}>0, \\
C=-c_{22}>0, \quad D=b_{11} c_{22}>0 .
\end{gathered}
$$

Case $1\left(\tau_{1}=\tau_{2}=0\right)$. Equation (7) becomes

$$
\lambda^{2}+(B+C) \lambda+A+D=0
$$

Obviously, $B+C>0$. Therefore, the roots of (9) must have negative real parts if condition $\left(H_{3}\right): A+D>0$ holds. Namely, the positive equilibrium $E_{*}\left(x_{*}, y_{*}\right)$ is locally stable if $\left(H_{3}\right): A+D>0$ holds.

Case $2\left(\tau_{1}>0, \tau_{2}=0\right)$. Equation ( 7 ) can be transformed into the following form:

$$
\lambda^{2}+C \lambda+A+(B \lambda+D) e^{-\lambda \tau_{1}}=0
$$

Let $\lambda=i \omega_{1}\left(\omega_{1}>0\right)$ be a root of $(10)$; then we get

$$
\begin{gathered}
D \sin \tau_{1} \omega_{1}-B \omega_{1} \cos \tau_{1} \omega_{1}=C \omega_{1}, \\
D \cos \tau_{1} \omega_{1}+B \omega_{1} \sin \tau_{1} \omega_{1}=\omega_{1}^{2}-A .
\end{gathered}
$$

From (11), we can have

$$
\omega_{1}^{4}+\left(C^{2}-B^{2}-2 A\right) \omega_{1}^{2}+A^{2}-D^{2}=0 .
$$

It is easy to know that (12) has only one positive real root

$$
=\sqrt{\frac{-\left(C^{2}-B^{2}-2 A\right)+\sqrt{\left(C^{2}-B^{2}-2 A\right)^{2}-4\left(A^{2}-D^{2}\right)}}{2}},
$$

if $\left(H_{4}\right): A^{2}-D^{2}<0$ holds.

The corresponding critical value of time delay $\tau_{1 k}$ is

$$
\tau_{1 k}=\frac{1}{\omega_{10}} \arccos \frac{(D-B C) \omega_{10}^{2}-A D}{B^{2} \omega_{10}^{2}+D^{2}}+\frac{2 k \pi}{\omega_{10}}, \quad k=0,1,2 \ldots
$$

Next, we verify the transversality condition. Differentiating (10) with respect to $\tau_{1}$, we have

$$
\left[\frac{d \lambda}{d \tau_{1}}\right]^{-1}=-\frac{2 \lambda+C}{\lambda\left(\lambda^{2}+C \lambda+A\right)}+\frac{B}{\lambda(D+B \lambda)}-\frac{\tau_{1}}{\lambda} .
$$


Thus,

$$
\operatorname{Re}\left[\frac{d \lambda}{d \tau_{1}}\right]_{\tau_{1}=\tau_{10}}^{-1}=\frac{2 \omega_{10}^{2}+C^{2}-B^{2}-2 A}{B^{2} \omega_{10}^{2}+D^{2}}
$$

From (13) and (16), it is easy to verify that $\operatorname{Re}\left[d \lambda / d \tau_{1}\right]_{\tau_{1}=\tau_{10}}^{-1}>0$. Thus, the transversality condition is satisfied. In conclusion, we have the following results.

Theorem 1. If condition $\left(H_{4}\right): A^{2}-D^{2}<0$ holds, then the positive equilibrium $E_{*}\left(x_{*}, y_{*}\right)$ of system (2) is asymptotically stable for $\tau_{1} \in\left[0, \tau_{10}\right)$ and unstable when $\tau_{1}>\tau_{10} ;$ system (2) undergoes Hopf bifurcation at $\tau_{1}=\tau_{10}$.

Case $3\left(\tau_{1}=0, \tau_{2}>0\right)$. Equation (7) becomes

$$
\lambda^{2}+B \lambda+A+(C \lambda+D) e^{-\lambda \tau_{2}}=0
$$

Let $\lambda=i \omega_{2}\left(\omega_{2}>0\right)$ be a root of $(17)$; then we get

$$
\begin{gathered}
D \sin \tau_{2} \omega_{2}-C \omega_{2} \cos \tau_{2} \omega_{2}=B \omega_{2}, \\
D \cos \tau_{2} \omega_{2}+C \omega_{2} \sin \tau_{2} \omega_{2}=\omega_{2}^{2}-A,
\end{gathered}
$$

which follows that

$$
\omega_{2}^{4}+\left(B^{2}-C^{2}-2 A\right) \omega_{2}^{2}+A^{2}-D^{2}=0 .
$$

Similar to Case 1, we can know that (19) has only one positive root

$$
=\sqrt{\frac{-\left(B^{2}-C^{2}-2 A\right)+\sqrt{\left(B^{2}-C^{2}-2 A\right)^{2}-4\left(A^{2}-D^{2}\right)}}{2}}
$$

if $\left(H_{4}\right): A^{2}-D^{2}<0$ holds. The corresponding critical value of time delay $\tau_{2 k}$ is

$$
\tau_{2 k}=\frac{1}{\omega_{20}} \arccos \frac{(D-B C) \omega_{20}^{2}-A D}{C^{2} \omega_{20}^{2}+D^{2}}+\frac{2 k \pi}{\omega_{20}}, \quad k=0,1,2 \ldots
$$

Similar to Case 2, we can get $\operatorname{Re}\left[d \lambda / d \tau_{2}\right]_{\tau_{2}=\tau_{20}}^{-1}>0$. That is, the transversality condition is satisfied. Therefore, we have the following results.

Theorem 2. If condition $\left(\mathrm{H}_{4}\right): A^{2}-D^{2}<0$ holds, then the positive equilibrium $E_{*}\left(x_{*}, y_{*}\right)$ of system (2) is asymptotically stable for $\tau_{2} \in\left[0, \tau_{20}\right)$ and unstable when $\tau_{2}>\tau_{20} ;$ system (2) undergoes Hopf bifurcation at $\tau_{2}=\tau_{20}$.

Case $4\left(\tau_{1}>0, \tau_{2} \in\left(0, \tau_{20}\right)\right)$. We consider (7) with $\tau_{2}$ in its stable interval, regarding $\tau_{1}$ as a parameter.
Let $\lambda=i \omega(\omega>0)$ be a root of (7). Separating real and imaginary parts, then we get

$$
\begin{aligned}
& \left(B \omega-D \sin \tau_{2} \omega\right) \cos \tau_{1} \omega-D \cos \tau_{2} \omega \sin \tau_{1} \omega \\
& \quad=-C \omega \cos \tau_{2} \omega, \\
& \left(B \omega-D \sin \tau_{2} \omega\right) \sin \tau_{1} \omega+D \cos \tau_{2} \omega \cos \tau_{1} \omega \\
& \quad=\omega^{2}-A-C \omega \sin \tau_{2} \omega,
\end{aligned}
$$

which follows that

$$
\begin{aligned}
\omega^{4} & +\left(C^{2}-B^{2}-2 A\right) \omega^{2}+A^{2}-D^{2}-2 \\
& \times\left(C \omega^{3}-(A C+B D) \omega\right) \sin \tau_{2} \omega=0 .
\end{aligned}
$$

Suppose the following. $\left(H_{5}\right)$ : equation (23) has at least finite positive roots. And all the roots of (23) are denoted by $\omega_{1}, \omega_{2}, \ldots, \omega_{n}$. For every fixed $\omega_{i}(i=1,2, \ldots, n)$, the corresponding critical value of time delay $\left\{\tau_{1_{i}}^{(j)} \mid j=\right.$ $0,1,2, \ldots\}$ is

$$
\begin{gathered}
\tau_{1_{i}}^{(j)}=\frac{1}{\omega_{i}} \arccos \frac{\left[(D-B C) \omega_{i}^{2}-A D\right] \cos \tau_{2} \omega_{i}}{B^{2} \omega_{i}^{2}+D^{2}-2 B D \omega_{i} \sin \tau_{2} \omega_{i}}+\frac{2 j \pi}{\omega_{i}}, \\
i=1,2, \ldots n, \quad j=0,1,2, \ldots
\end{gathered}
$$

Let $\tau_{10}^{*}=\min \left\{\tau_{1_{i}}{ }^{(j)} \mid i=1,2, \ldots n, j=0,1,2, \ldots\right\}, \omega_{10}^{*}=\omega_{1_{i 0}}$. Differentiating (7) with respect to $\tau_{1}$, we obtain

$$
\begin{aligned}
{\left[\frac{d \lambda}{d \tau_{1}}\right]^{-1}=} & -\frac{2 \lambda+B e^{-\lambda \tau_{1}}+C e^{-\lambda \tau_{2}}\left(1-\tau_{2} \lambda\right)-\tau_{2} D e^{-\lambda\left(\tau_{1}+\tau_{2}\right)}}{\lambda\left(\lambda^{2}+C \lambda+A\right)} \\
& -\frac{\tau_{1}}{\lambda} .
\end{aligned}
$$

Thus,

$$
\operatorname{Re}\left[\frac{d \lambda}{d \tau_{1}}\right]_{\tau_{1}=\tau_{10}^{*}}^{-1}=\frac{\Delta_{1} \Delta_{3}+\Delta_{2} \Delta_{4}}{\Delta_{3}^{2}+\Delta_{4}^{2}}
$$

where

$$
\begin{aligned}
\Delta_{1}= & C \cos \tau_{2} \omega_{10}^{*}-\tau_{2} C \omega_{10}^{*} \sin \tau_{2} \omega_{10}^{*} \\
& +\tau_{2} D \sin \tau_{2} \omega_{10}^{*} \sin \tau_{10}^{*} \omega_{10}^{*} \\
& +\left(B-\tau_{2} D \cos \tau_{2} \omega_{10}^{*}\right) \cos \tau_{10}^{*} \omega_{10}^{*}, \\
\Delta_{2}= & 2 \omega_{10}^{*}-C \sin \tau_{2} \omega_{10}^{*}-\tau_{2} C \omega_{10}^{*} \cos \tau_{2} \omega_{10}^{*} \\
& +\tau_{2} D \sin \tau_{2} \omega_{10}^{*} \cos \tau_{10}^{*} \omega_{10}^{*} \\
& -\left(B-\tau_{2} D \cos \tau_{2} \omega_{10}^{*}\right) \sin \tau_{10}^{*} \omega_{10}^{*}, \\
\Delta_{3}= & C\left(\omega_{10}^{*}\right)^{2} \cos \tau_{2} \omega_{10}^{*}, \\
\Delta_{4}= & \left(\omega_{10}^{*}\right)^{3}-A \omega_{10}^{*}-C\left(\omega_{10}^{*}\right)^{2} \sin \tau_{2} \omega_{10}^{*} .
\end{aligned}
$$


Obviously, if condition $\left(H_{6}\right): \Delta_{1} \Delta_{3}+\Delta_{2} \Delta_{4} \neq 0$, then the transversality condition is satisfied. Therefore, we have the following results.

Theorem 3. If conditions $\left(H_{5}\right)$ and $\left(H_{6}\right)$ hold and $\tau_{2} \in$ $\left(\tau_{20}, 0\right)$, then the positive equilibrium $E_{*}\left(x_{*}, y_{*}\right)$ of system (2) is asymptotically stable for $\tau_{1} \in\left[0, \tau_{10}^{*}\right)$ and unstable when $\tau_{1}>\tau_{10}^{*}$; system (2) undergoes Hopf bifurcation at $\tau_{1}=\tau_{10}^{*}$.

Case $5\left(\tau_{2}>0, \tau_{1} \in\left(0, \tau_{10}\right)\right)$. We consider (7) with $\tau_{1}$ in its stable interval, regarding $\tau_{2}$ as a parameter.

Let $\lambda=i \omega(\omega>0)$ be a root of (7). Separating real and imaginary parts, then we get

$$
\begin{aligned}
& \left(C \omega-D \sin \tau_{1} \omega\right) \cos \tau_{2} \omega-D \cos \tau_{1} \omega \sin \tau_{2} \omega \\
& \quad=-B \omega \cos \tau_{1} \omega, \\
& \left(C \omega-D \sin \tau_{1} \omega\right) \sin \tau_{2} \omega+D \cos \tau_{1} \omega \cos \tau_{2} \omega \\
& =\omega^{2}-A-B \omega \sin \tau_{1} \omega,
\end{aligned}
$$

which follows that

$$
\begin{aligned}
\omega^{4} & +\left(B^{2}-C^{2}-2 A\right) \omega^{2}+A^{2}-D^{2} \\
& -2\left(B \omega^{3}-(A B+C D) \omega\right) \sin \tau_{1} \omega=0 .
\end{aligned}
$$

Similar to Case 4 , suppose the following. $\left(H_{7}\right)$ : equation (23) has at least finite positive roots, which are denoted by $\omega_{1}, \omega_{2}, \ldots, \omega_{n}$. The corresponding critical value of time delay for every $\omega_{i}$ is

$$
\begin{gathered}
\tau_{2_{i}}^{(j)}=\frac{1}{\omega_{2 i}} \arccos \frac{\left[(D-B C) \omega_{2 i}^{2}-A D\right] \cos \tau_{1} \omega_{2 i}}{C^{2} \omega_{2 i}^{2}+D^{2}-2 C D \omega_{2 i} \sin \tau_{1} \omega_{2 i}}+\frac{2 j \pi}{\omega_{2 i}}, \\
i=1,2, \ldots, n, \quad j=0,1,2, \ldots
\end{gathered}
$$

Let $\tau_{20}^{*}=\min \left\{\tau_{2_{i}}^{(j)} \mid i=1,2, \ldots, n, j=0,1,2, \ldots\right\}, \omega_{20}^{*}=\omega_{2_{i 0}}$.

Next, we give the following assumption:

$$
\left(H_{8}\right):\left[\frac{d \operatorname{Re}(\lambda)}{d \tau_{2}}\right]_{\tau_{2}=\tau_{20}^{*}}^{-1} \neq 0 .
$$

Hence, we have the following theorem.

Theorem 4. If conditions $\left(H_{7}\right)$ and $\left(H_{8}\right)$ hold and $\tau_{1} \in$ $\left[0, \tau_{10}\right)$, the positive equilibrium $E_{*}\left(x_{*}, y_{*}\right)$ of system (2) is asymptotically stable for $\tau_{2} \in\left[0, \tau_{20}^{*}\right)$ and unstable when $\tau_{2}>$ $\tau_{20}^{*}$; system (2) undergoes Hopf bifurcation at $\tau_{2}=\tau_{20}^{*}$.

\section{Direction and Stability of the Hopf Bifurcation}

In this section, we will use normal form theory and center manifold argument introduced by Hassard et al. [12] to determine the direction of Hopf bifurcation and stability of the bifurcating periodic solutions of system (2) with respect to $\tau_{1}$ for $\tau_{2} \in\left(0, \tau_{20}\right)$. Without loss of generality, we assume that $\tau_{2}^{*}<\tau_{10}^{*}$, where $\tau_{2}^{*} \in\left(0, \tau_{20}\right)$.

Let $\tau_{1}=\tau_{10}^{*}+\mu, \mu \in R$. Then $\mu=0$ is the Hopf bifurcation value of system (2). Rescaling the time $t \rightarrow t / \tau_{1}$, then system (2) can be written as

$$
\dot{u}(t)=L_{\mu} u_{t}+F\left(\mu, u_{t}\right),
$$

where

$$
\begin{gathered}
L_{\mu} \phi=\left(\tau_{10}^{*}+\mu\right)\left(A^{\prime} \phi(0)+C^{\prime} \phi\left(-\frac{\tau_{2}^{*}}{\tau_{10}^{*}}\right)+B^{\prime} \phi(-1)\right), \\
F\left(\mu, u_{t}\right)=\left(\tau_{10}^{*}+\mu\right)\left(F_{1}, F_{2}\right)^{T},
\end{gathered}
$$

with

$$
\begin{gathered}
\phi(\theta)=\left(\phi_{1}(\theta), \phi_{2}(\theta)\right)^{T} \in C\left([-1,0], R^{2}\right), \\
A^{\prime}=\left(\begin{array}{cc}
0 & a_{12} \\
a_{21} & 0
\end{array}\right), \quad B^{\prime}=\left(\begin{array}{cc}
b_{11} & 0 \\
0 & 0
\end{array}\right), \\
C^{\prime}=\left(\begin{array}{cc}
0 & 0 \\
0 & c_{22}
\end{array}\right), \\
F_{1}=a_{13} \phi_{1}(0) \phi_{2}(0)+a_{14} \phi_{1}(0) \phi_{1}(-1), \\
F_{2}=a_{23} \phi_{1}(0) \phi_{2}(0)+a_{24} \phi_{2}(0) \phi_{2}\left(-\frac{\tau_{2}^{*}}{\tau_{10}^{*}}\right) .
\end{gathered}
$$

By the Riesz representation theorem, there exists a $2 \times 2$ matrix function $\eta(\theta, \mu):[-1,0] \rightarrow R^{2}$ whose elements are of bounded variation, such that

$$
L_{\mu} \phi=\int_{-1}^{0} d \eta(\theta, \mu) \phi(\theta), \quad \phi \in C\left([-1,0], R^{2}\right) .
$$

In fact, we can choose

$$
\eta(\theta, \mu)= \begin{cases}\left(\tau_{10}^{*}+\mu\right)\left(A^{\prime}+B^{\prime}+C^{\prime}\right), & \theta=0, \\ \left(\tau_{10}^{*}+\mu\right)\left(B^{\prime}+C^{\prime}\right), & \theta \in\left[-\frac{\tau_{2}^{*}}{\tau_{10}^{*}}, 0\right), \\ \left(\tau_{10}^{*}+\mu\right) B^{\prime}, & \theta \in\left(-1,-\frac{\tau_{2}^{*}}{\tau_{10}^{*}}\right), \\ 0, & \theta=-1 .\end{cases}
$$

For $\phi \in C\left([-1,0], R^{2}\right)$, we define

$$
\begin{gathered}
A(\mu) \phi= \begin{cases}\frac{d \phi(\theta)}{d \theta}, & -1 \leq \theta<0, \\
\int_{-1}^{0} d \eta(\theta, \mu) \phi(\theta), & \theta=0,\end{cases} \\
R(\mu) \phi= \begin{cases}0, & -1 \leq \theta<0, \\
F(\mu, \phi), & \theta=0 .\end{cases}
\end{gathered}
$$

Then system (32) is equivalent to the following operator equation:

$$
\dot{u}(t)=A(\mu) u_{t}+R(\mu) u_{t} .
$$


The adjoint operator $A^{*}$ of $A$ is defined by

$$
A^{*}(\mu) \phi= \begin{cases}-\frac{d \varphi(s)}{d s}, & 0<s \leq 1, \\ \int_{-1}^{0} d \eta^{T}(s, \mu) \varphi(-s), & s=0,\end{cases}
$$

associated with a bilinear form

$$
\begin{aligned}
\langle\varphi, \phi\rangle= & \bar{\varphi}(0) \phi(0) \\
& -\int_{\theta=-1}^{0} \int_{\xi=0}^{\theta} \bar{\varphi}(\xi-\theta) d \eta(\theta) \phi(\xi) d \xi,
\end{aligned}
$$

where $\eta(\theta)=\eta(\theta, 0)$.

From the analysis above, we can see that $\pm i \tau_{10}^{*} \omega_{10}^{*}$ are the eigenvalues of $A(0)$ and they are also eigenvalues of $A^{*}(0)$. We assume that $\rho(\theta)=\left(1, \rho_{2}\right)^{T} e^{i \tau_{10}^{*} \omega_{10}^{*} \theta}$ are the eigenvectors of $A(0)$ belonging to the eigenvalue $+i \tau_{10}^{*} \omega_{10}^{*}$ and $\rho^{*}(\theta)=$ $D\left(1, \rho_{2}^{*}\right)^{T} e^{i \tau_{10}^{*} \omega_{10}^{*} s}$ are the eigenvectors of $A^{*}(0)$ belonging to the eigenvalue $-i \tau_{10}^{*} \omega_{10}^{*}$.

By a simple computation, we can obtain

$$
\begin{gathered}
\rho_{2}=\frac{a_{21}}{i \omega_{10}^{*}-c_{22} e^{-i \tau_{2}^{*} \omega_{10}^{*}}}, \\
\rho_{2}^{*}=-\frac{i \omega_{10}^{*}+b_{11} e^{-i \tau_{10}^{*} \omega_{10}^{*}}}{a_{21}} .
\end{gathered}
$$

In addition, from (40), we can get

$$
D=\left[1+\rho_{2} \bar{\rho}_{2}^{*}+b_{11} e^{-i \tau_{10}^{*} \omega_{10}^{*}}+c_{22} e^{-i \tau_{2}^{*} \omega_{10}^{*}} \rho_{2} \bar{\rho}_{2}^{*}\right]^{-1},
$$

such that $\left\langle q^{*}, q\right\rangle=1$ and $\left\langle q^{*}, \bar{q}\right\rangle=0$.

Next, we get the following coefficients by using a similar computation process to [13]:

$$
\begin{gathered}
g_{20}=2 \tau_{10}^{*} \bar{D}\left[a_{13} q^{(2)}(0)+a_{14} q^{(1)}(-1)\right. \\
\left.+\bar{\rho}_{2}^{*}\left(a_{23} q^{(2)}(0)+a_{24} q^{(2)}(0) q^{(2)}\left(-\frac{\tau_{2}^{*}}{\tau_{10}^{*}}\right)\right)\right], \\
g_{11}=\tau_{10}^{*} \bar{D}\left[a_{13}\left(\bar{q}^{(2)}(0)+q^{(2)}(0)\right)\right. \\
+a_{14}\left(\bar{q}^{(1)}(-1)+q^{(1)}(-1)\right) \\
+\bar{\rho}_{2}^{*}\left(a_{23}\left(\bar{q}^{(2)}(0)+q^{(2)}(0)\right)\right. \\
+a_{24}\left(q^{(2)}(0) \bar{q}^{(2)}\left(-\frac{\tau_{2}^{*}}{\tau_{10}^{*}}\right)\right. \\
\left.\left.\left.+\bar{q}^{(2)}(0) q^{(2)}\left(-\frac{\tau_{2}^{*}}{\tau_{10}^{*}}\right)\right)\right)\right],
\end{gathered}
$$

$$
\begin{aligned}
& g_{02}=2 \tau_{10}^{*} \bar{D}\left[a_{13} \bar{q}^{(2)}(0)+a_{14} \bar{q}^{(1)}(-1)\right. \\
& \left.+\bar{\rho}_{2}^{*}\left(a_{23} \bar{q}^{(2)}(0)+a_{24} \bar{q}^{(2)}(0) \bar{q}^{(2)}\left(-\frac{\tau_{2}^{*}}{\tau_{10}^{*}}\right)\right)\right], \\
& g_{21}=2 \tau_{10}^{*} \bar{D}\left[a _ { 1 3 } \left(W_{11}^{(1)}(0) q^{(2)}(0)+\frac{1}{2} W_{20}^{(1)}(0) \bar{q}^{(2)}(0)\right.\right. \\
& \left.+W_{11}^{(2)}(0)+\frac{1}{2} W_{20}^{(2)}(0)\right) \\
& +a_{14}\left(W_{11}^{(1)}(0) q^{(1)}(-1)+\frac{1}{2} W_{20}^{(1)}(0) \bar{q}^{(1)}(-1)\right. \\
& \left.+W_{11}^{(1)}(-1)+\frac{1}{2} W_{20}^{(1)}(-1)\right) \\
& +\bar{\rho}_{2}^{*}\left(a _ { 2 3 } \left(W_{11}^{(1)}(0) q^{(2)}(0)+\frac{1}{2} W_{20}^{(1)}(0) \bar{q}^{(2)}(0)\right.\right. \\
& \left.+W_{11}^{(2)}(0)+\frac{1}{2} W_{20}^{(2)}(0)\right) \\
& +a_{24}\left(W_{11}^{(2)}(0) q^{(2)}\left(-\frac{\tau_{2}^{*}}{\tau_{10}^{*}}\right)\right. \\
& +\frac{1}{2} W_{20}^{(2)}(0) \bar{q}^{(2)}\left(-\frac{\tau_{2}^{*}}{\tau_{10}^{*}}\right) \\
& +W_{11}^{(2)}\left(-\frac{\tau_{2}^{*}}{\tau_{10}^{*}}\right) q^{(2)}(0) \\
& \left.\left.\left.+\frac{1}{2} W_{20}^{(2)}\left(-\frac{\tau_{2}^{*}}{\tau_{10}^{*}}\right) \bar{q}^{(2)}(0)\right)\right)\right] \text {, }
\end{aligned}
$$

with

$$
\begin{aligned}
W_{20}(\theta)= & \frac{i g_{20} q(0)}{\tau_{10}^{*} \omega_{10}^{*}} e^{i \tau_{10}^{*} \omega_{10}^{*} \theta} \\
& +\frac{i \bar{g}_{02} \bar{q}(0)}{3 \tau_{10}^{*} \omega_{10}^{*}} e^{-i \tau_{10}^{*} \omega_{10}^{*} \theta}+E_{1} e^{2 i \tau_{10}^{*} \omega_{10}^{*} \theta}, \\
W_{11}(\theta)=- & \frac{i g_{11} q(0)}{\tau_{10}^{*} \omega_{10}^{*}} e^{i \tau_{10}^{*} \omega_{10}^{*} \theta} \\
& +\frac{i \bar{g}_{11} \bar{q}(0)}{\tau_{10}^{*} \omega_{10}^{*}} e^{-i \tau_{10}^{*} \omega_{10}^{*} \theta}+E_{2} .
\end{aligned}
$$

And $E_{1}, E_{2}$ satisfy the following equations, respectively:

$$
\begin{aligned}
\left(\begin{array}{cc}
2 i \omega_{10}^{*}-b_{11} e^{-2 i \tau_{10}^{*} \omega_{10}^{*}} & -a_{12} \\
-a_{21} & 2 i \omega_{10}^{*}-c_{22} e^{-2 i \tau_{2}^{*} \omega_{10}^{*}}
\end{array}\right) E_{1} \\
=2\left(\begin{array}{l}
G_{11} \\
G_{21}
\end{array}\right), \\
\left(\begin{array}{ll}
b_{11} & a_{12} \\
a_{21} & c_{22}
\end{array}\right) E_{2}=-\left(\begin{array}{l}
H_{11} \\
H_{21}
\end{array}\right),
\end{aligned}
$$



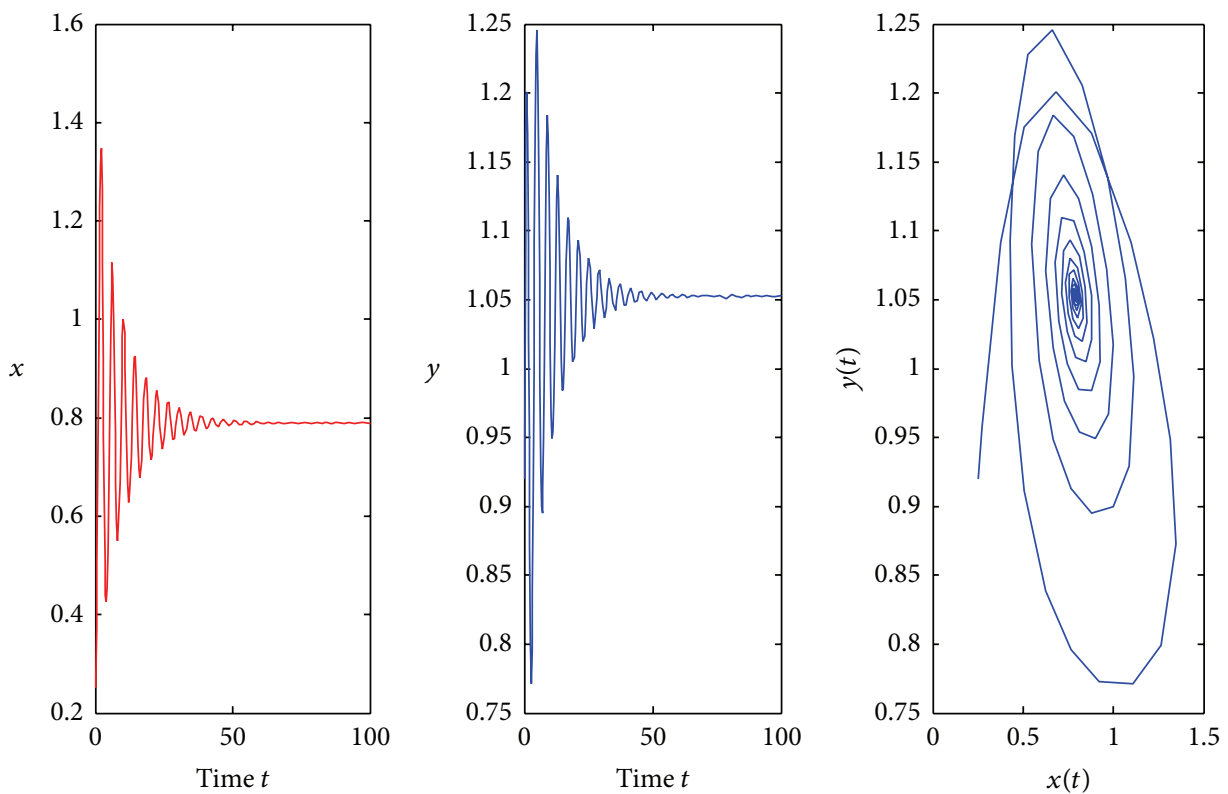

$-x$

$-y$

FIGURE 1: $E_{*}$ is asymptotically stable for $\tau_{1}=0.95<\tau_{10}=1.0241$ with initial values " $0.25 ; 0.92$."
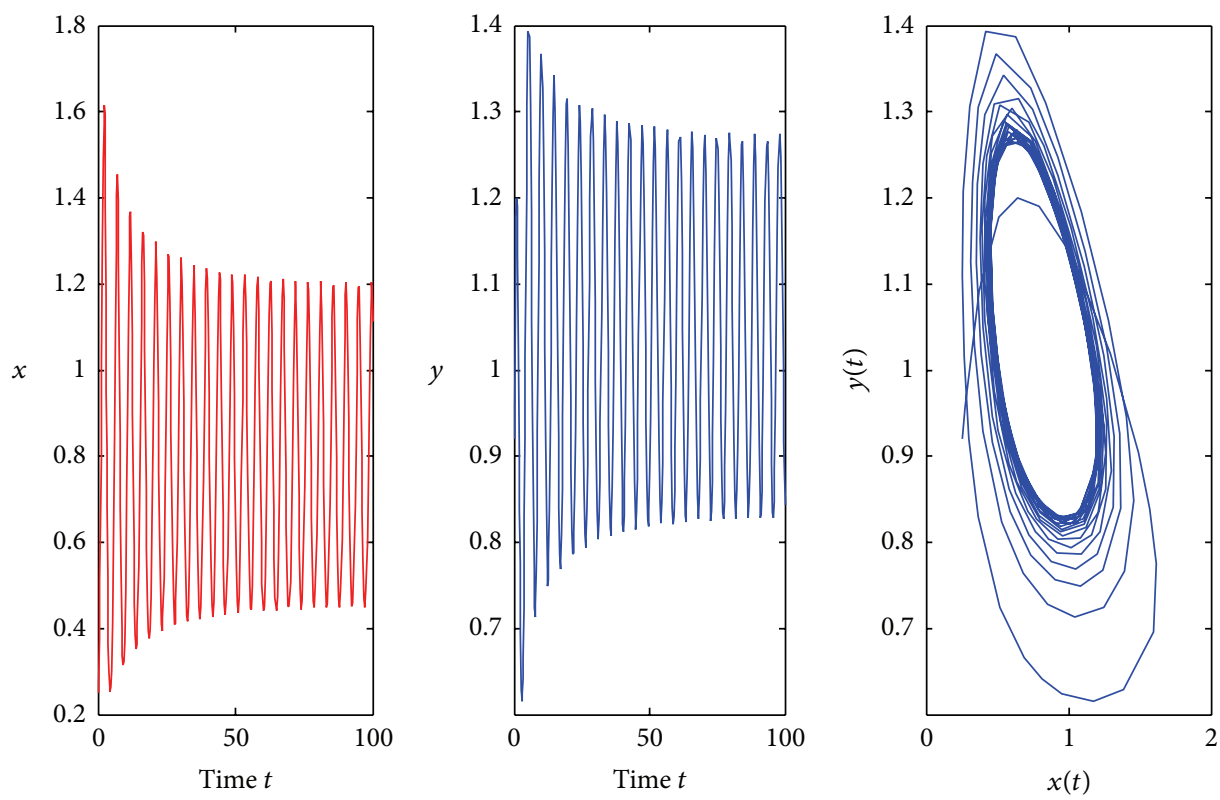

- $x \quad-y$

FIgURE 2: $E_{*}$ is unstable for $\tau_{1}=1.10>\tau_{10}=1.0241$ with initial values " $0.25 ; 0.92$."

with

$$
\begin{gathered}
G_{11}=a_{13} q^{(2)}(0)+a_{14} q^{(1)}(-1) \\
G_{21}=a_{23} q^{(2)}(0)+a_{24} q^{(2)}(0) q^{(2)}\left(-\frac{\tau_{2}^{*}}{\tau_{10}^{*}}\right) \\
H_{11}=a_{13}\left(\bar{q}^{(2)}(0)+q^{(2)}(0)\right) \\
+a_{14}\left(\bar{q}^{(1)}(-1)+q^{(1)}(-1)\right)
\end{gathered}
$$

$$
\begin{aligned}
H_{21}= & a_{23}\left(\bar{q}^{(2)}(0)+q^{(2)}(0)\right) \\
& +a_{24}\left(q^{(2)}(0) \bar{q}^{(2)}\left(-\frac{\tau_{2}^{*}}{\tau_{10}^{*}}\right)+\bar{q}^{(2)}(0) q^{(2)}\left(-\frac{\tau_{2}^{*}}{\tau_{10}^{*}}\right)\right) .
\end{aligned}
$$

Then, we can get the following coefficients:

$$
C_{1}(0)=\frac{i}{2 \tau_{10}^{*} \omega_{10}^{*}}\left(g_{11} g_{20}-2\left|g_{11}\right|^{2}-\frac{\left|g_{02}\right|^{2}}{3}\right)+\frac{g_{21}}{2}
$$



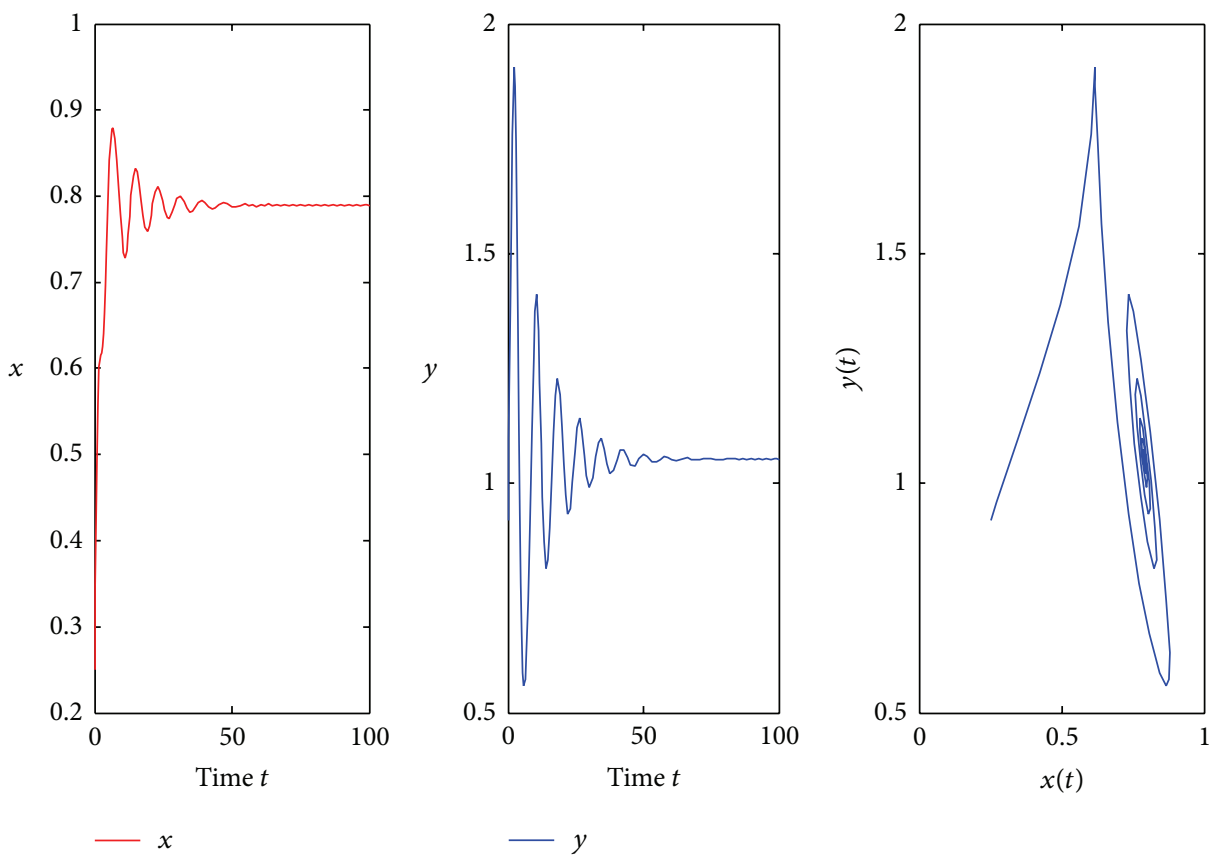

FigURE 3: $E_{*}$ is asymptotically stable for $\tau_{2}=1.65<\tau_{20}=1.8631$ with initial values " $0.25 ; 0.92$."
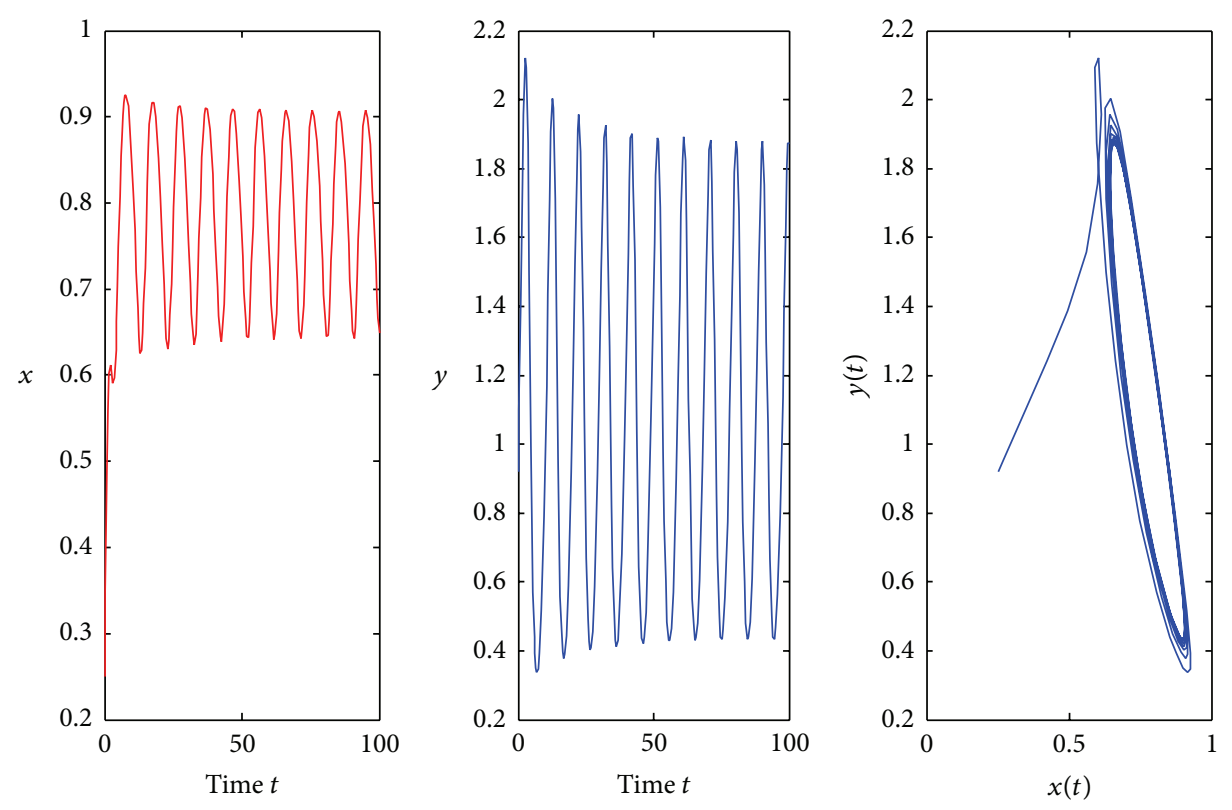

FIGURE 4: $E_{*}$ is unstable for $\tau_{2}=2.05>\tau_{20}=1.8631$ with initial values " $0.25 ; 0.92$."

$$
\begin{gathered}
\mu_{2}=-\frac{\operatorname{Re}\left\{C_{1}(0)\right\}}{\operatorname{Re}\left\{\lambda^{\prime}\left(\tau_{10}^{*}\right)\right\}}, \\
\beta_{2}=2 \operatorname{Re}\left\{C_{1}(0)\right\}, \\
T_{2}=-\frac{\operatorname{Im}\left\{C_{1}(0)\right\}+\mu_{2} \operatorname{Im}\left\{\lambda^{\prime}\left(\tau_{10}^{*}\right)\right\}}{\tau_{10}^{*} \omega_{10}^{*}} .
\end{gathered}
$$

Theorem 5. If $\mu_{2}>0\left(\mu_{2}<0\right)$, then the Hopf bifurcation is supercritical (subcritical); if $\beta_{2}<0\left(\beta_{2}>0\right)$, then the bifurcating periodic solutions are stable (unstable); if $T_{2}>$ $0\left(T_{2}<0\right)$, then the period of the bifurcating periodic solution increases (decreases).

\section{Numerical Simulation}

In conclusion, we have the following results for system (2).

In this section, some numerical simulations were given to support the analytical results obtained in the previous 

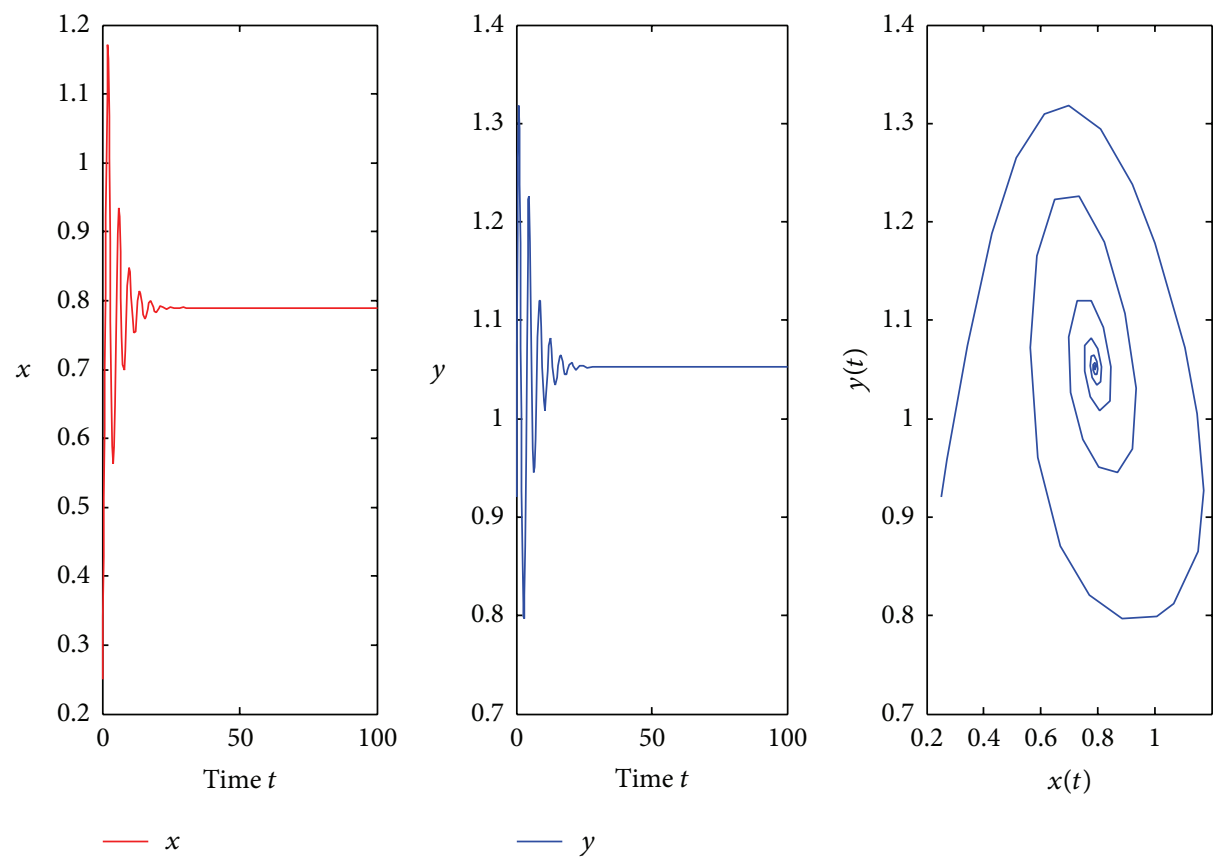

FIGURE 5: $E_{*}$ is asymptotically stable for $\tau_{2}=0.5, \tau_{1}=0.86<\tau_{10}^{*}=1.0931$ with initial values " $0.25 ; 0.92$."
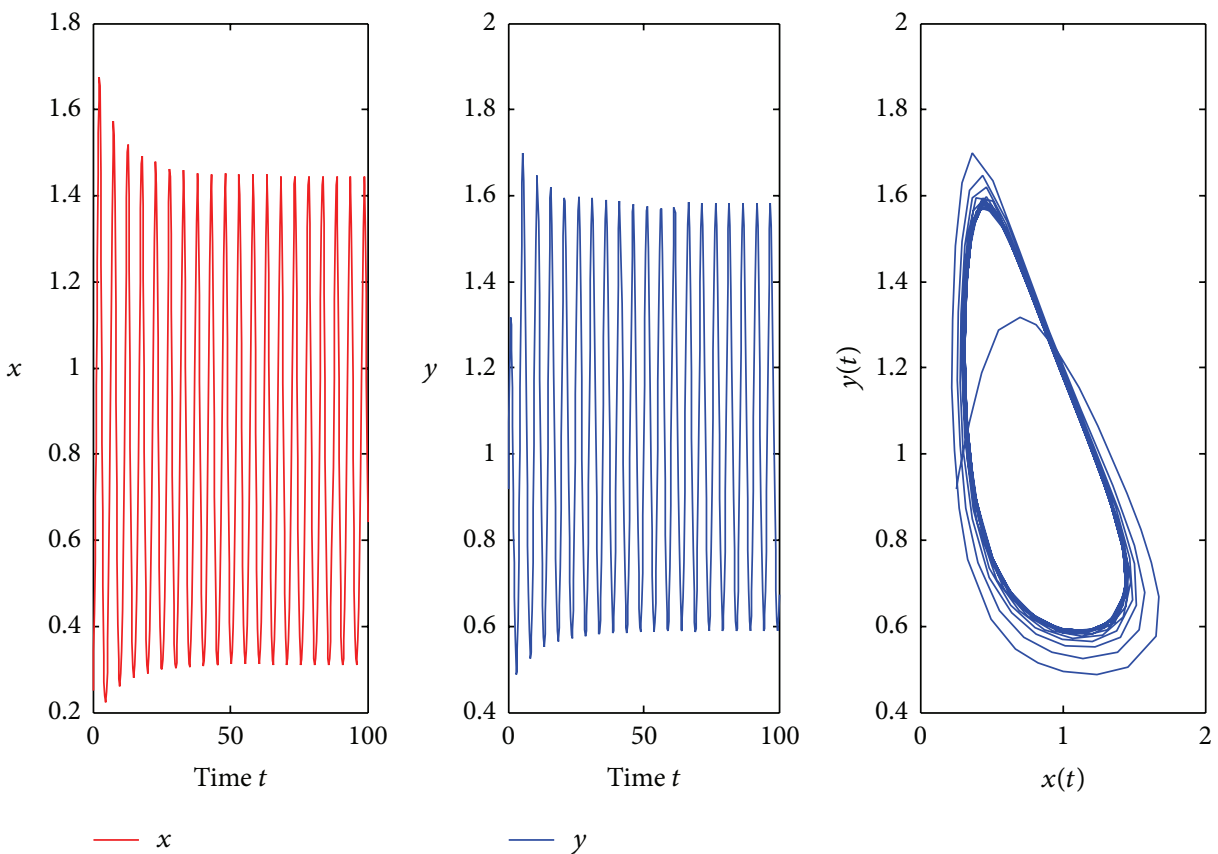

FigURE 6: $E_{*}$ is unstable for $\tau_{2}=0.5, \tau_{1}=1.15>\tau_{10}^{*}=1.0931$ with initial values "0.25;0.92."

sections. Let $r_{1}=2, r_{2}=1.5, N_{1}=1, N_{2}=2, \sigma_{12}=0.4$, and $\sigma_{21}=0.6$; then we have the following system:

$$
\begin{gathered}
\frac{d x(t)}{d t}=2 x(t)\left[1-x\left(t-\tau_{1}\right)-0.2 y(t)\right], \\
\frac{d y(t)}{d t}=1.5 y(t)\left[1-\frac{y\left(t-\tau_{2}\right)}{2}-0.6 x(t)\right] .
\end{gathered}
$$

Obviously, conditions $\left(H_{1}\right)$ and $\left(H_{2}\right)$ hold. By a simple calculation, we obtain that (48) has a unique positive equilibrium $E_{*}(0.7894,1.0526)$. Then we have $A+D=0.9471>0$. Namely, condition $\left(\mathrm{H}_{3}\right)$ holds.

Firstly, by computation we can easily get $A^{2}-D^{2}=$ $-1.4637<0$; that is, condition $\left(H_{4}\right)$ holds. Further, we have $\omega_{10}=1.4150, \tau_{10}=1.0241$. By Theorem 1 , we obtain the corresponding waveform and the phase plots are shown in 

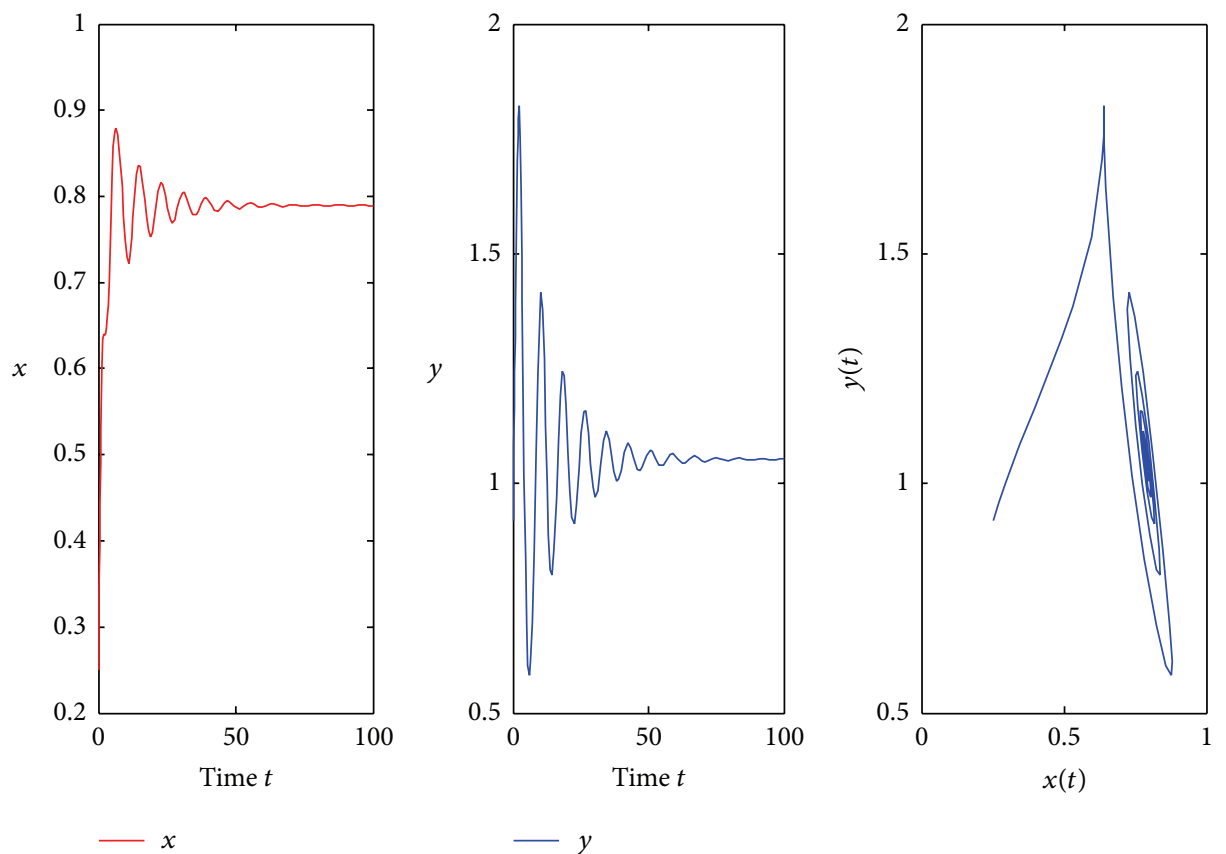

FIGURE $7: E_{*}$ is asymptotically stable for $\tau_{1}=0.15, \tau_{2}=1.65<\tau_{20}^{*}=1.8062$ with initial values " $0.25 ; 0.92$."
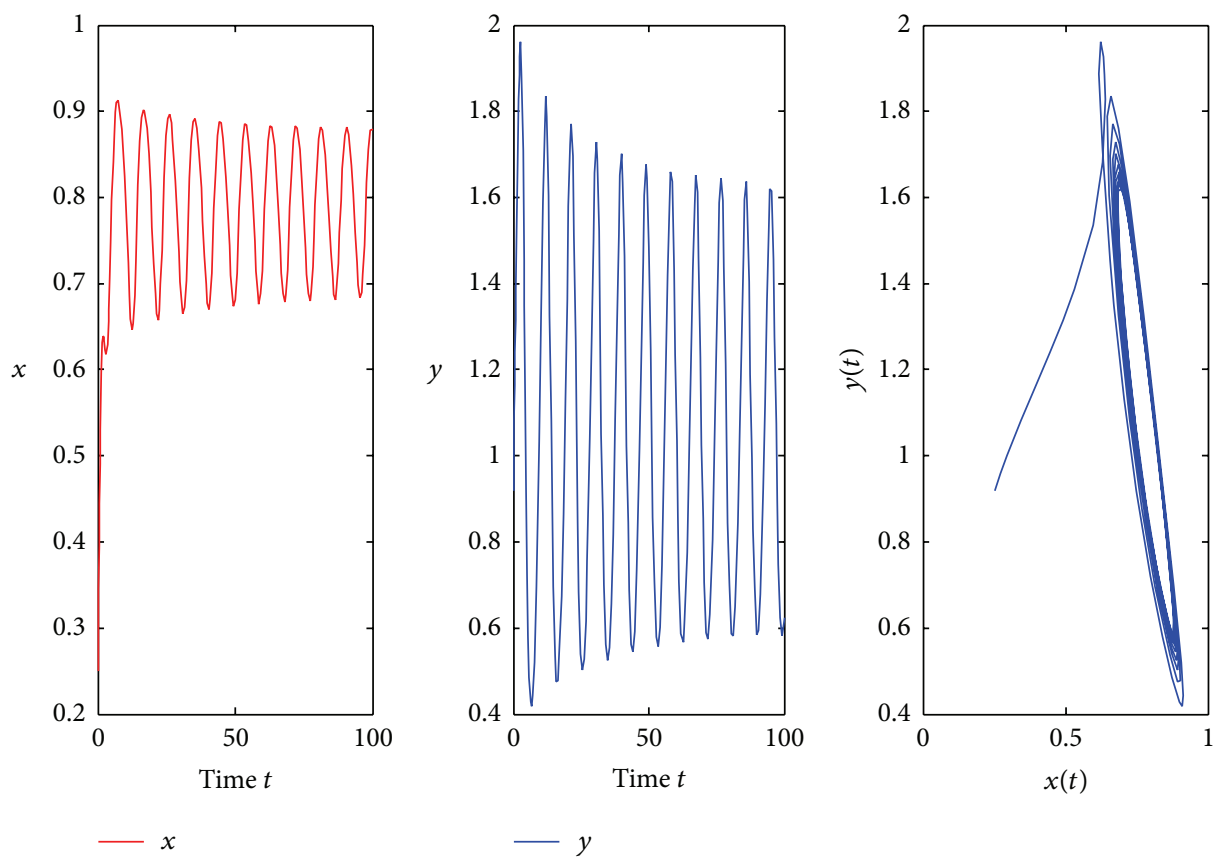

FIGURE 8: $E_{*}$ is unstable for $\tau_{1}=0.15, \tau_{2}=1.95>\tau_{20}^{*}=1.8062$ with initial values " $0.25 ; 0.92 . "$

Figures 1 and 2. Similarly, we have $\omega_{20}=0.7029, \tau_{20}=1.8631$. From Theorem 2, we obtain the corresponding waveform and the phase plots are shown in Figures 3 and 4.

Secondly, we can obtain $\omega_{10}^{*}=1.3398, \tau_{10}^{*}=1.0931$ when $\tau_{1}>0, \tau_{2}=0.5 \in\left(0, \tau_{20}\right)$. From Theorem 3, we know that if we let $\tau_{2}$ be in its stable interval and regard $\tau_{1}$ as a parameter, then $E_{*}(0.7894,1.0526)$ is asymptotically stable for $\tau_{1} \in\left[0, \tau_{10}^{*}\right)$ and unstable when $\tau_{1}>\tau_{10}^{*}$ and a Hopf bifurcation occurs. This is can be illustrated by
Figures 5 and 6 . In addition, we have $\lambda^{\prime}\left(\tau_{10}^{*}\right)=-0.0221-$ $0.9139 i$ and from $(47)$ we get $C_{1}(0)=-0.4057+1.9027 i, \mu_{2}=$ $-18.3575<0, \beta_{2}=-0.8114<0$, and $T_{2}=12.7546>0$. From Theorem 5 we know that the direction of the Hopf bifurcation is subcritical and the bifurcating periodic solutions are stable.

Lastly, for $\tau_{2}>0, \tau_{1}=0.15 \in\left(0, \tau_{10}\right)$, we have $\omega_{20}^{*}=$ $0.7119, \tau_{20}^{*}=1.8062$. That is, when $\tau_{2}$ increases from zero to $\tau_{20}^{*}=1.8062, E_{*}(0.7894,1.0526)$ is asymptotically stable. Then it will loses its stability and a Hopf bifurcation occurs 
once $\tau_{2}>\tau_{20}^{*}=1.8062$. This property can be illustrated by Figures 7 and 8 .

\section{Conclusion}

A competitive model of market structure with consumptive delays is studied in this paper. By analyzing the distribution of the roots of the associated characteristic equation, we obtained the conditions for the local stability of the model and the existence of the Hopf bifurcation. The main results are given in Theorems 1-4, which show that the consumptive delays play important roles in the model. It is proved that when some conditions are satisfied, then Hopf bifurcation occurs when the delay passes through the corresponding critical value. In reality, the occurrence of Hopf bifurcation means that the coexistence of the two products in system (2) changes from the positive equilibrium to a limit cycle, which is not welcome in reality. Furthermore, the explicit formulas determining the stability and the direction of the bifurcating periodic solutions are given by using the normal form theory and center manifold argument. The main results are given in Theorem 5. From the view of economy, if the bifurcating periodic solutions are stable, the two products of the same type may coexist in an oscillatory mode. This is valuable from the view of economics. However, our study is restricted only to the theoretical analysis of such economic phenomena. It may be helpful for field investigation or experimental studies on the real situation.

\section{Conflict of Interests}

The author declares that there is no conflict of interests regarding the publication of this paper.

\section{Acknowledgment}

The author is grateful to the referees for their valuable comments and suggestions on the paper.

\section{References}

[1] G. Stépán, "Great delay in a predator-prey model," Nonlinear Analysis: Theory, Methods \& Applications, vol. 10, no. 9, pp. 913929, 1986.

[2] K. Wang and Y. Zhu, "Global attractivity of positive periodic solution for a Volterra model," Applied Mathematics and Computation, vol. 203, no. 2, pp. 493-501, 2008.

[3] Y. Lv and Z. Du, "Existence and global attractivity of a positive periodic solution to a Lotka-Volterra model with mutual interference and Holling III type functional response," Nonlinear Analysis: Real World Applications, vol. 12, no. 6, pp. 3654-3664, 2011.

[4] X. Wang, Z. Du, and J. Liang, "Existence and global attractivity of positive periodic solution to a Lotka-Volterra model," Nonlinear Analysis: Real World Applications, vol. 11, no. 5, pp. 40544061, 2010.

[5] F. Chen, "The permanence and global attractivity of LotkaVolterra competition system with feedback controls," Nonlinear Analysis: Real World Applications, vol. 7, no. 1, pp. 133-143, 2006.
[6] Y. Song, M. Han, and Y. Peng, "Stability and Hopf bifurcations in a competitive Lotka-Volterra system with two delays," Chaos, Solitons \& Fractals, vol. 22, no. 5, pp. 1139-1148, 2004.

[7] Z. Teng, "On the persistence and positive periodic solution for planar competing Lotka-Volterra systems," Annals of Differential Equations. Weifen Fangcheng Niankan, vol. 13, no. 3, pp. 275286, 1997.

[8] J. A. Brander and M. S. Taylor, "The simple economics of easter Island: a Ricardo-Malthus model of renewable resource use," American Economic Review, vol. 88, no. 1, pp. 119-138, 1998.

[9] D. Delfino and P. J. Simmons, "Infectious disease and economic growth: the case of tuberculosis," Working Paper, 1999.

[10] J. D. Farmer, "A simple model for the non equilibrium dynamics and evolution of financial market," International Journal of Theoretical and Applied Finance, vol. 3, no. 3, pp. 425-441, 2000.

[11] D. M. Kong, "Evolution of market structure under LotkaVolterra system," Journal of Industrial Engineering and Engineering Management, vol. 19, no. 3, pp. 77-81, 2005 (Chinese).

[12] B. D. Hassard, N. D. Kazarinoff, and Y. H. Wan, Theory and Applications of Hopf Bifurcation, Cambridge University Press, Cambridge, UK, 1981.

[13] M. Liao, X. Tang, and C. Xu, "Bifurcation analysis for a threespecies predator-prey system with two delays," Communications in Nonlinear Science and Numerical Simulation, vol. 17, no. 1, pp. 183-194, 2012. 


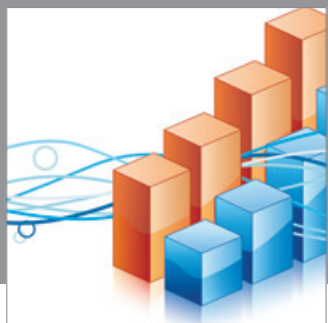

Advances in

Operations Research

mansans

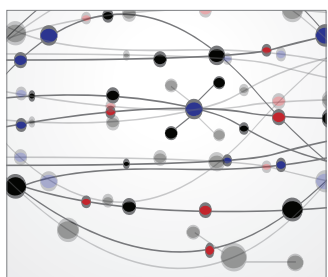

The Scientific World Journal
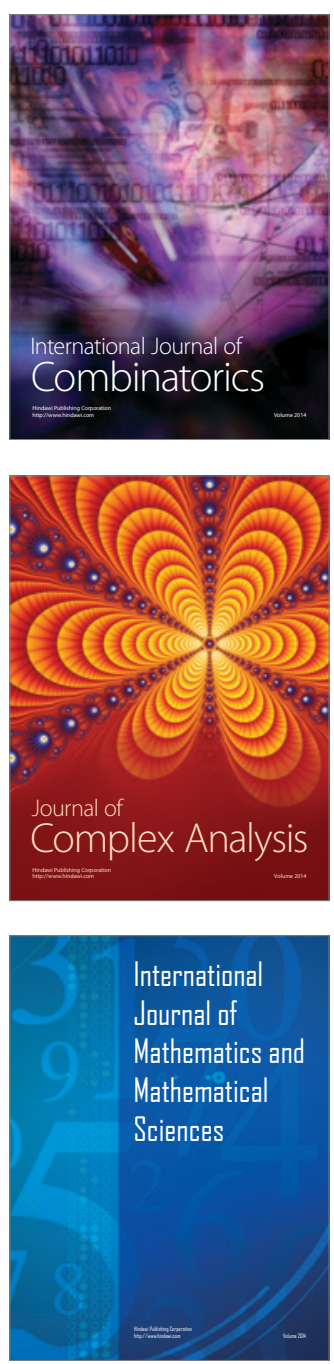
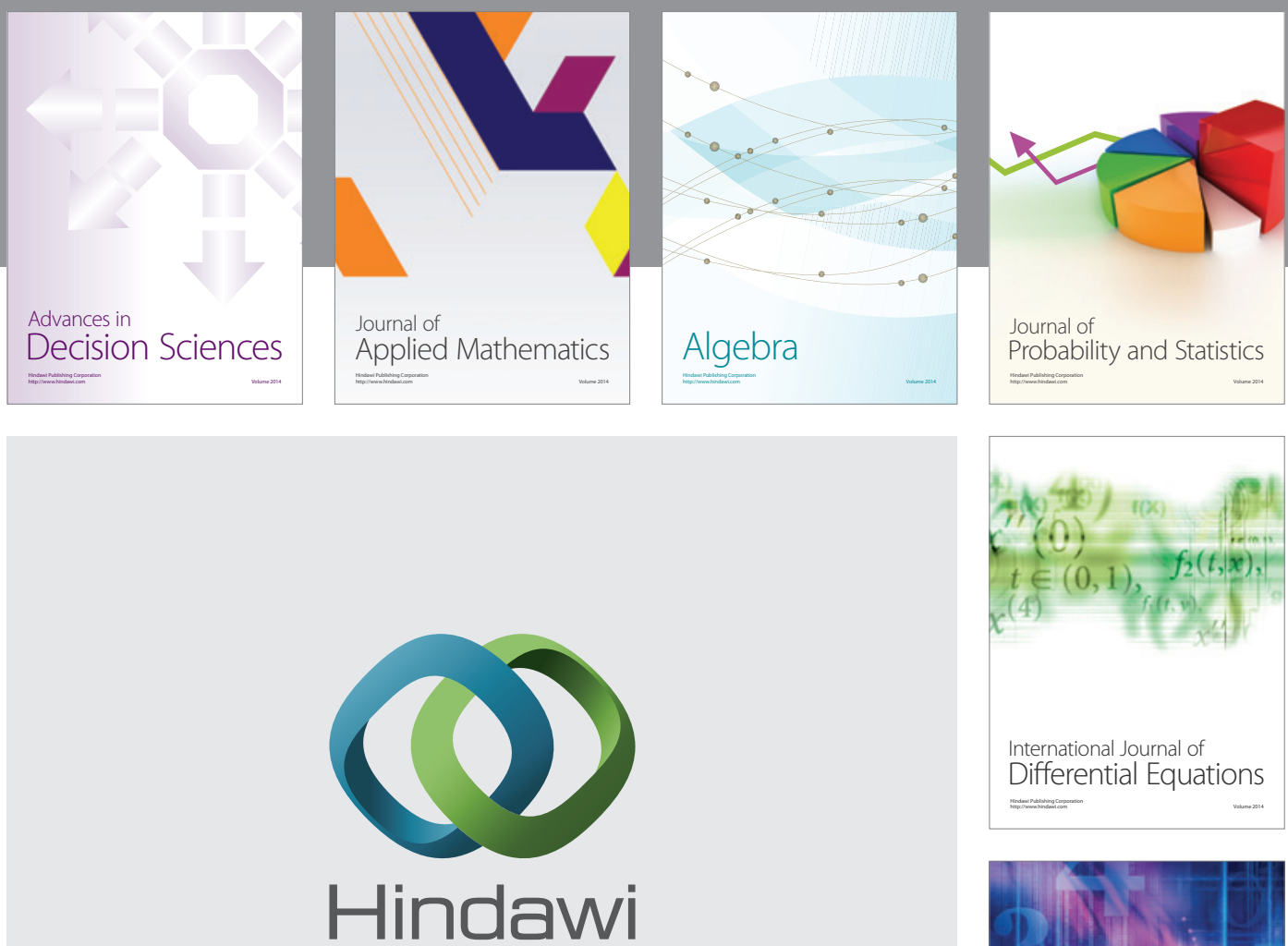

Submit your manuscripts at http://www.hindawi.com
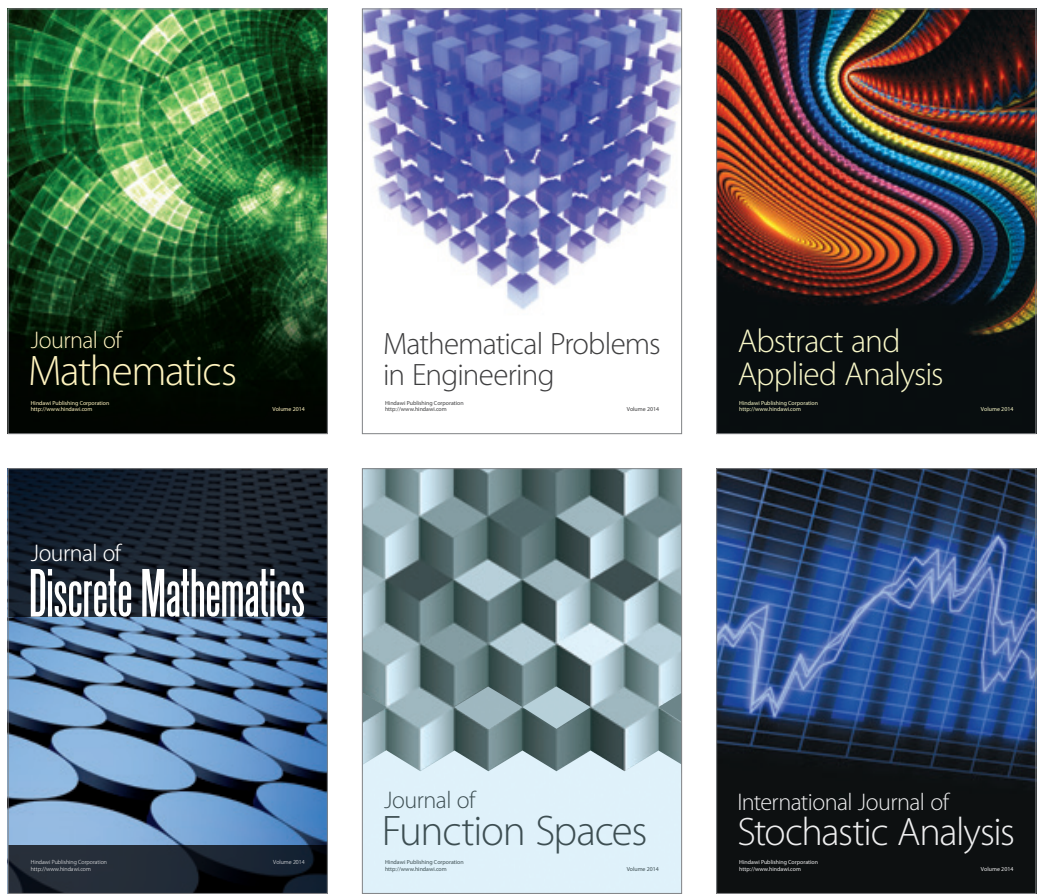

Journal of

Function Spaces

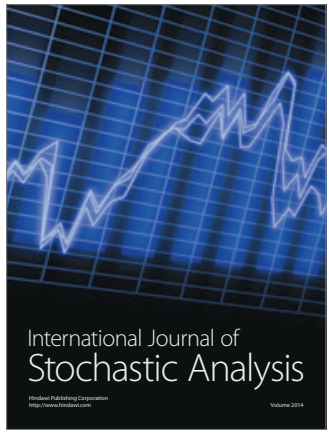

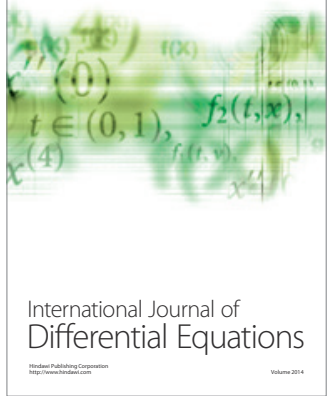
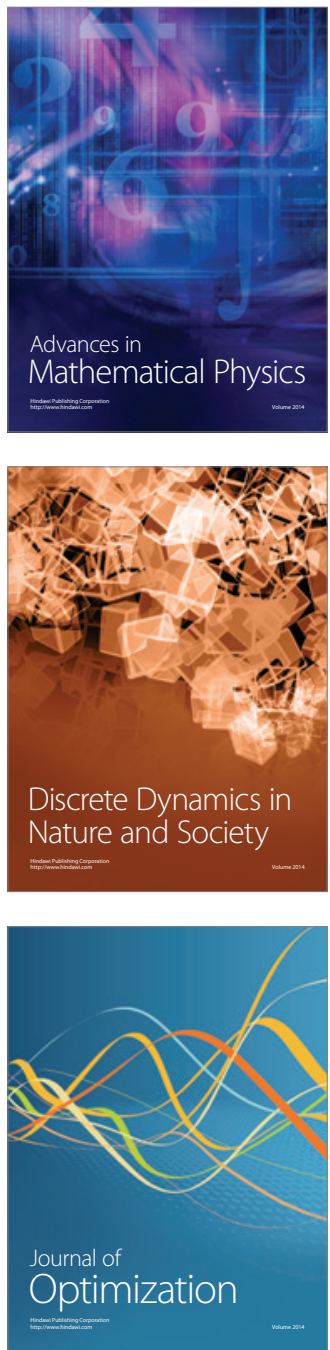